\title{
Role of protein adsorption on haemodialysis-induced complement activation and neutrophil defects
}

\author{
P. Neveceral, M. Markert and J.-P. Wauters \\ Division of Nephrology and Laboratory of Clinical Chemistry, University Hospital, Lausanne, Switzerland
}

\begin{abstract}
The present clinical study investigated the role of protein adsorption on complement activation and neutrophil functions during in vivo haemodialysis. The parameters were measured simultaneously at the arterial and venous sites of a cuprophan (CU) dialyser with or without pretreatment with human albumin, human immunoglobulins or human total plasma proteins (PLP). Leukocyte count, complement activation (C3a des arg), oxygen radical production and chemotaxis were measured at time zero and $15 \mathrm{~min}$ at the arterial and venous sites of the dialyser. Leukopenia observed at both sites was prevented only with PLP treatment. Complement activation was maximal at the venous site, but was not prevented by any of the treatments. Neutrophil oxygen radical production and chemotaxis were significantly decreased only at the venous site and restored to normal with any of the three treatments. Complement activation was maximal at the venous site, but was not prevented by any of the treatments. Protein adsorption on the dialyser membrane seems to modulate the bioincompatibility parameters in a different way. Depending on the functions tested, the protein fractions have different protecting effects, indicating the multifactorial mechanism implicated in the CU haemodialysis-induced leukopenia, complement activation and neutrophil defect.
\end{abstract}

Key words: biocompatibility; complement; leukopenia; haemodialysis; neutrophil function

\section{Introduction}

The transient leukopenia and neutrophil dysfunctions occurring at the initiation of haemodialysis on cellulosic membranes have been mostly attributed to complement activation [1-4]. However a discrepancy between the degree of complement activation, leukopenia and neutrophil dysfunctions at the maximum of leukopenia was reported $[5,6]$. These defects have not been evidenced on synthetic membranes or after reuse

Correspondence and offprint requests to: Professor J. P. Wauters, Division of Nephrology, CHUV, CH-1011 Lausanne, Switzerland. of cellulosic membranes [7,8]. Moreover, neutrophil defects were shown to be altered after $15 \mathrm{~min}$ of dialysis only at the venous site of the cuprophan (CU) dialyser, suggesting that the membrane contact itself could play a role [9]. In addition adsorption of proteins on the membrane surface seemed to interfere with these phenomena. Indeed, in vitro experiments showed a different recovery of proteins which was by far the largest on polysulphone (PS) followed by polyacrilonitrile (PAN) and cuprophane (CU). In contrast the recovery of proteins on polycarbonate (PC) was almost negligible and this differential protein coating was found to alter the neutrophil functional response [10].

Since the most marked changes in white blood cell count, complement activation and neutrophil dysfunctions were observed on the CU dialyser, we initiated a clinical study of leukopenia, complement activation and neutrophil functions measured simultaneously at the arterial and venous sites with and without pretreatment of the dialyser with human albumin (ALB), human immunoglobulin (IG) or human total plasma proteins (PLP) on the CU membrane.

\section{Subjects and methods}

\section{Patients and procedure}

Sixteen patients aged 30-75 years (mean 60), on chronic haemodialysis for a mean of 48 months (range 6-181), entered the study. The dialyser consisted of CF 15-11 (Travenol) made of CU. The CU membrane was either used in its original set-up (five patients), or pretreated with a solution of PLP (four patients), ALB (four patients) or IG (three patients). The protein solutions used were diluted with saline from inititial concentrated solutions ( $20 \%$, Red Cross, Bern) to concentrations of $5 \%$ for PLP, $4 \%$ for ALB and $4 \%$ for IG. The coating procedure with the protein solutions was initiated by flushing the dialyser with 11 saline. Two hundred $\mathrm{ml}$ of the protein solution were then introduced into the blood compartment of the dialyser and left within the dialyser for $15 \mathrm{~min}$ at room temperature while no dialysate was running. After $15 \mathrm{~min}$ of contact within the dialyser, the protein solution was rinsed out with 11 saline. After this treatment, the clinical dialysis session was started with blood flow maintained at $250-300 \mathrm{ml} / \mathrm{min}$ while the dialysate, which contained bicarbonate, had a flow rate of $500 \mathrm{ml} / \mathrm{min}$ in a 
single-pass mode. Heparin was administered at individually adapted doses ranging from 2000 to $5000 \mathrm{U}$ at the start of dialysis and 500 to $750 \mathrm{U}$ hourly thereafter.

\section{Neutrophil preparation}

Three to $6 \mathrm{ml}$ blood samples (heparinized, $10 \mathrm{U} / \mathrm{ml}$ ) were obtained from the patients' arteriovenous fistulae immediately before initiation of dialysis (Time 0 ), and from arterial and venous sites of the dialyser $15 \mathrm{~min}$ after the onset of dialysis. Leukocytes were counted in the total blood on an automated analyser (Sysmex CC-800). Purified preparations of more than $95 \%$ neutrophils were obtained in a single centrifugation step on a discontinuous Percoll gradient (76\%/60\%, volume/volume) as already described [6]. The neutrophil population was identical whatever the group or the time of the dialysis.

\section{Oxygen radical determination}

Luminol- and lucigenin-enhanced chemiluminescence (CL) were used, the latter being a specific indicator of superoxide production and the former being dependent on hydrogen peroxide, other radicals and myeloperoxidase $[11,12]$. Appropriate stimuli were mixed with $5 \times 10^{4}$ neutrophils in a volume of $500 \mu \mathrm{l}$ of Hanks buffer supplemented with $56 \mu \mathrm{mol} / 1$ of luminol or $0.1 \mathrm{mmol} / 1$ of lucigenin at $37^{\circ} \mathrm{C}$, and the resulting light emission was measured in $\mathrm{mV}$ on a LKB luminometer at 5 min intervals until peak $C L$ was reached (20-30 $\mathrm{min})$, as described [7]. Opsonized zymosan (OZ) was prepared as in [7] and used at a final concentration of $1.5 \mathrm{mg} / \mathrm{ml}$.

\section{Chemotaxis}

Chemotaxis was assessed by the 'under agarose' method exactly as described [13]. Four series of two wells were cut in agarose which covered the bottom of culture dishes. The outer well received $5 \mu$ l of the chemotactic factor or medium and the inner well $5 \mu \mathrm{l}$ of the cell suspension containing $2 \times 10^{7}$ cells $/ \mathrm{ml}$. Zymosan-activated plasma (ZAP), containing $\mathrm{C} 5 \mathrm{a}$ des Arg, which was prepared according to [6], and formyl-methionyl-leucyl-phenylalanine (FMLP, $0.1 \mu \mathrm{mol} / \mathrm{l}$ ) were used as stimuli.

\section{Complement activation}

One $\mathrm{ml}$ blood sample obtained from the patients' arteriovenous fistula was taken in tubes containing EDTA at a final concentration of $10 \mathrm{mmol} / 1$ just before the start of dialysis and at $15 \mathrm{~min}$ at the arterial and venous site of the dialyser. Tubes were immediately centrifuged $\left(2000 \mathrm{~g}, 5 \mathrm{~min}, 4^{\circ} \mathrm{C}\right)$, then plasma was collected and frozen at $-80^{\circ} \mathrm{C}$ until analysis. $\mathrm{C} 3 \mathrm{a}$ des arg concentrations were measured by radioimmunoassay according to the manufacturer's instructions (Hans Rahn \& Co., Zürich, Dept Amersham).

\section{Statistical analysis}

Statistical analysis was performed using the paired $t$-test. Individual values are represented.

\section{Results}

Figure 1 illustrates the evolution of leukocyte counts at Time 0 and $15 \mathrm{~min}$ of dialysis at the arterial and venous sites. As already observed, no significant difference was evidenced between arterial and venous sites at $15 \mathrm{~min}$ of dialysis [9]. Pretreatment with total PLP corrected the leukopenia at the arterial site as well as at the venous site. In contrast neither a solution of ALB nor of IG prevented the leukocyte drop. This was not due to a non-adhesiveness of ALB or IG on the membrane. Indeed, after washing the proteincoated dialyser with a saline solution, these proteins were eluted from the dialyser in $200 \mathrm{ml}$ of a solution containing glycerol $(10 \%)$, mercapto-ethanol $(5 \%)$, sodium dodecyl sulfate (2\%) and Tris- $\mathrm{HCl}(0.0625 \mathrm{~mol}$, $\mathrm{pH}$ 6.8) and identified by sodium dodecylsulfate polyacrilamide gel electrophoresis (data not shown). The protein recovery of $0.3 \%$ for PLP, $0.5 \%$ for ALB and $0.8 \%$ for IG did not correlate with the initial amount introduced in the dialyser indicating that the type of protein and the selective reactivity of the membrane must play a role. Since an effect was observed at these amounts of proteins, no other concentration of protein was tested, except for ALB, which showed an identical leukopenia using a $2 \%$ or $4 \%$ solution.

Although C5a is known as a better activator of neutrophils than $\mathrm{C} 3 \mathrm{a}$, its great affinity for $\mathrm{C} 5 \mathrm{a}$ receptors and its rapid clearance makes it a less sensitive cumulative marker. Indeed, at $15 \mathrm{~min}$ of dialysis, C5a des arg was found to be less than $10 \mathrm{ng} / \mathrm{ml}$ at the arterial site, while it increased up to $53 \pm 15.4 \mathrm{ng} / \mathrm{ml}$ at the venous site. Complement activation was thus measured by the accumulation of C3a des arg and is shown in Figure 2. Whatever the pretreatment method, the C3a des arg levels were similar at $15 \mathrm{~min}$ to the levels measured on a new uncoated CU membrane. Moreover, the same significant differences were observed at $15 \mathrm{~min}$ between the arterial and venous site.

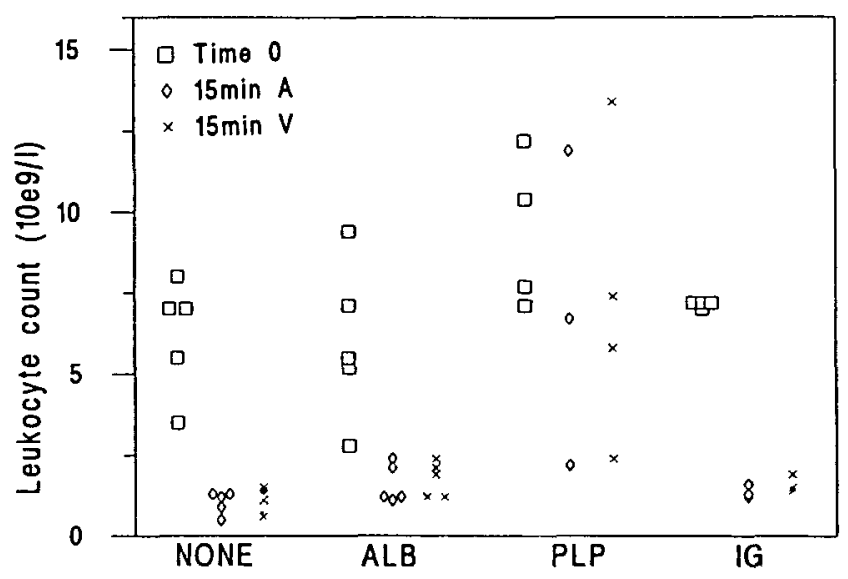

Fig. 1. Leukocyte count in patients at Time $0,15 \mathrm{~min}$ arterial site (A) and $15 \mathrm{~min}$ venous site (V) during dialysis on a new cuprophane membrane without treatment (NONE), pretreated with albumin (ALB), total plasma proteins (PLP) or immunoglobulins (IG)) as described under Methods. 


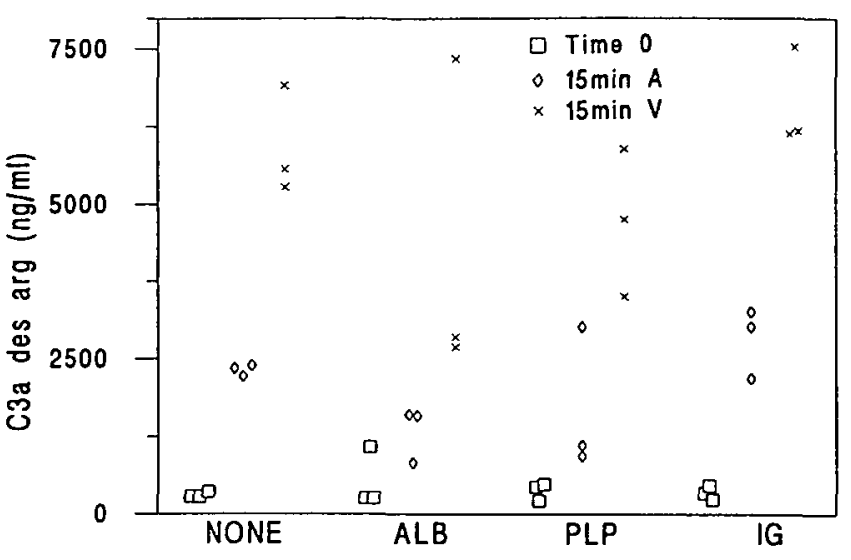

Fig. 2. Complement activation as measured by the C3a des arg levels on a new CU or CU pretreated with ALB, PLP or IG, at Time 0, $15 \mathrm{~min}$ arterial site and $15 \mathrm{~min}$ venous site. Legends and abbreviations are as in Fig. 1.

Figure 3 shows the effect of different pretreatments on oxygen radical production when stimulated with $\mathrm{OZ}$ by neutrophils collected at $0 \mathrm{~min}$ and $15 \mathrm{~min}$ at the arterial and venous site of the dialyser. Whether luminol- (Figure 3a) or lucigenin- (Figure 3b)
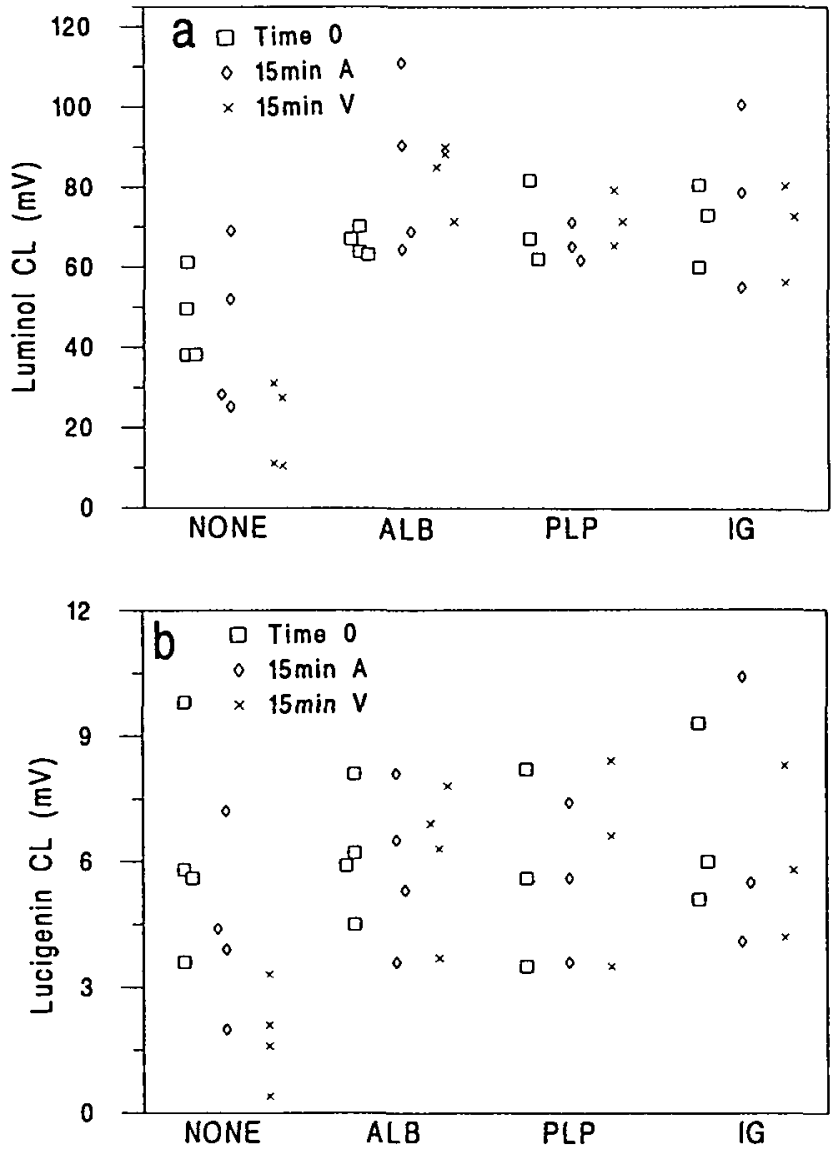

Fig. 3. Luminol- (a) and lucigenin- (b) amplified chemiluminescence (CL) of neutrophils stimulated with opsonized zymosan on a new CU or CU pretreated with ALB, PLP or IG. Legends and abbreviations are as in Fig. 1. enhanced $\mathrm{CL}$ was measured, coating the CU membrane with either a solution of ALB, PLP or IG significantly corrected the defect observed at the venous site $(P<0.05)$ of a new untreated membrane. When other stimuli were used, such as phorbol myristate acetate or FMLP, the defect observed at the venous site of the dialyser was also corrected upon membrane pretreatment (data not shown).
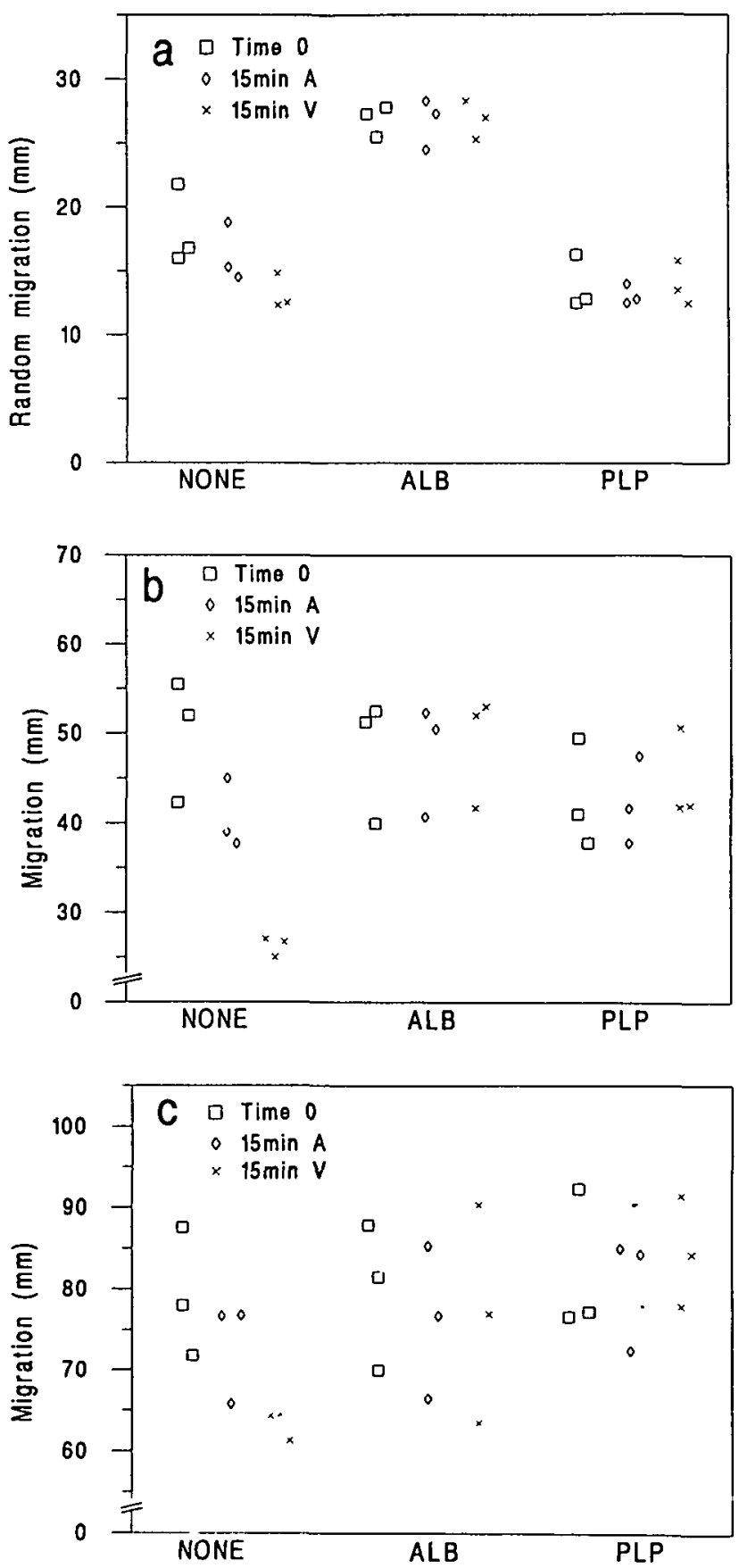

Fig. 4. Random migration (a), migration stimulated with zymosanactivated plasma (b) and FMLP (c) by neutrophils of patients dialysed on CU membrane without treatment, or treated with ALB or PLP. Legends and abbreviations are as in Fig. 1. 
Chemotaxis, random and stimulated with ZAP or FMLP, is shown in Figures $4 a, 4 b$, and $4 c$, respectively. Again, a depressed response $(P<0.05)$ was seen only in cells collected at the venous site of a new untreated $\mathrm{CU}$ membrane. Treatment with ALB or PLP was able to correct this decreased response. A significant difference was observed between the random migration of neutrophils in the ALB group compared to the PLP group $(P<0.05)$. However, the random migration of neutrophils measured in the three groups was within the normal range determined in 21 healthy controls (10-33 mm).

\section{Discussion}

Leukopenia, complement activation with elevation of the anaphylatoxin $\mathrm{C} 3 \mathrm{a}$ des arg and neutrophil dysfunctions occur on a CU dialyser. These effects are attenuated with the dialyser reuse as documented by others and our earlier studies $[7,14,15]$. Furthermore, despite a similar leukopenia at both venous and arterial sides, the neutrophil functional alterations are evidenced only after passage through the dialyser as we previously reported [9]. The neutrophil dysfunctions observed during dialysis have been attributed to downregulation of the cells by activated complement resulting from the passage of the blood through the dialyser membrane [9]. However the present study shows that upon pretreatment of CU with ALB or IG, the defects observed in neutrophil functions at the venous site of the dialyser were abolished while leukopenia and complement activation were not modified. In contrast, pretreatment of CU membrane with PLP corrected both leukopenia and neutrophil dysfunctions, but not complement activation. Furthermore, at the maximum of complement activation and leukopenia, neutrophils isolated at the arterial site were still functional, despite higher accumulation of $\mathrm{C} 3 \mathrm{a}$ des arg at the venous site as early as $1 \mathrm{~min}[16,17]$. The dissociation between the various alterations is thus suggestive of complementdependent and complement-independent pathways modulating leukopenia and neutrophil functions; other proteins and the type of membrane must play an additional role [9]. Indeed, in vitro studies have shown that protein coating of cellulosic or synthetic membranes with plasma did either stimulate or depress neutrophil oxidative metabolism according to the type of membrane [10], while coating the membranes with albumin only depressed neutrophil oxidative metabolism [17].

It was also recently reported that upon pretreatment with albumin $25 \%$, elastase accumulation was decreased while complement activation was not modified [18]. In addition, accumulation of granulocyte lactoferrin and myeloperoxidase in the plasma measured at the arterial site was not modified with reuse despite a marked attenuation of complement activation, while the rise in elastase was decreased with reuse [18].

These discrepant results about the protective effect of protein coating on complement activation, leukopenia and neutrophil functions indicate that different protein solutions are not sufficient to confer a similar protection as plasma. This suggests that a specific competitive and selective adsorption among different types of proteins could confer these various protective effects.

In conclusion, the present study confirms that complement activation is not the only possible pathway leading to leukopenia and neutrophil functional alterations on a cuprophan membrane. Selective protein adsorption on the dialyser membrane interferes differently on several parameters of membrane bioincompatibility: the nature of the membrane, the direct neutrophil contact with the membrane, the protein adsorption and as yet unknown factors could each play a role, alone or in synergy.

\section{References}

1. Craddock PR, Fehr J, Dalmasso AP, Brigham KL, Jacob HS. Hemodialysis leukopenia: pulmonary vascular leukostasis resulting from complement activation by dialyzer cellophan membranes. J Clin Invest 1977; 59: 879-888

2. Chenoweth DE. Complement activation during hemodialysis: clinical observations, proposed mechanisms and theoretical implications. Artif Organs 1984; 8: 281-287

3. Wissow LS, Greenbery RS, Burns RO, Osafsky SG, Gutman RA, Baker PJ. Alterated leukocyte chemiluminescence during hemodialysis. Clin Immunol 1981; 1: 262-265

4. Markert M, Waridel PA, Heierli C, Frei J, Wauters JP. Neutrophil functions during hemodialysis. Contrib Nephrol 1988; 62: $99-108$

5. Aljama P, Bird PA, Ward MK, Tanboga H, Sheridan T, Craig H, Kerr DN. Hemodialysis-induced leukopenia and activation of complement: effects of different membranes. Proc Eur Dial Transplant Assoc 1978; 15: 144-153

6. Heierli C, Markert M, Lambert PH, Kuwahara T, Wauters JP. On the mechanism of hemodialysis induced neutropenia: a study with 5 new and re-used membranes. Nephrol Dial Transplant 1988; 3: 773-783

7. Markert M, Heierli C, Kuwahara T, Frei J, Wauters JP. Dialyzer polymorphonuclear neutrophil oxidative metabolism during dialysis: a comparative study with 5 new and re-used membranes. Clin Nephrol 1988; 29: 129-136

8. Kuwahara T, Markert M, Wauters JP. Biocompatibility aspects of dialyzer reprocessing: a comparison of 3 re-use methods and 3 membranes. Clin Nephrol 1989; 32: 139-143

9. Neveceral P, Markert M, Wauters JP. Neutrophil behaviour during hemodialysis: role of membrane contact. Trans Am Soc Artif Intern Organs 1988; XXXIV: 564-567

10. Kuwahara T, Markert M, Wauters JP. Proteins adsorbed on hemodialysis membranes modulate neutrophil activation. Artif Organs 1989; 13: 427-431

11. Gyllenhamer $H$. Lucigenin chemiluminescence in the assessment of neutrophil superoxide production. J Immunol Meth 1987; 87: 209-213

12. De Chatelet LR, Long GD, Shirley PS, Bass DA, Thomas MJ, Henderson FW, Cohen MS. Mechanism of the luminoldependent chemiluminescence of human neutrophils. $J$ Immunol 1987; 129: 1589-1593

13. Nelson RD, Quie PG, Simmons RL. Chemotaxis under agarose: a new and simple method for measuring chemotaxis and spontaneous migration of human polymorphonuclear leukocytes and monocytes. J Immunol 1975; 115: 1650-1656

14. Hakim RM, Lowrie EG. Effect of dialyzer reuse on leukopenia, hypoxemia and total hemolytic complement system. ASAIO Trans 1980; 26: 159-164 
15. Cheung AK, Henderson LW. Effect of complement activation by hemodialysis membranes. Am J Nephrol 1986; 6: $81-91$

16. Chenoweth DE, Cheung AK, Henderson LW. Anaphylatoxin formation during hemodialysis: effect of different dialyzer membranes. Kidney Int 1983; 24: 764-769
17. Markert M, Wauters JP. New aspects on oxidative metabolism of neutrophils during hemodialysis on different dialyzer membranes. Adv Exp Med Biol 1991; 297: 207-213

18. Hörl WH, Feinstein EI, Frischmuth IV, Gösele A, Massry SG. Plasma levels of main granulocyte components during hemodialysis. Am J Nephrol 1990; 10: 53-57

Received for publication: 10.1.94

Accepted in revised form: 14.10 .94 